\title{
Fístula de líquido cefalorraquídeo del receso lateral de seno esfenoidal. ¿Es el canal de Sternberg un defecto real?
}

\section{Cerebrospinal fluid leak of the lateral recess of the sphenoid sinus. Is Sternberg's channel a real defect?}

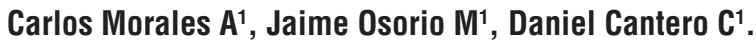

\begin{abstract}
RESUMEN
Las fístulas de líquido cefalorraquídeo (LCR) corresponden a una comunicación anómala entre el espacio subaracnoideo y la cavidad nasal. El origen de las fístulas laterales del seno esfenoidal se encuentra en un defecto congénito de la base del cráneo, con una incompleta o prematura fusión de los componentes óseos implicados en el complejo proceso de osificación del esfenoides. Ello origina un canal sin cobertura ósea, solo cubierto por tejido conectivo, denominado canal craneofaríngeo lateral o de Sternberg. Este es un punto débil donde pueden aparecer encefaloceles y/o fístulas, su ubicación lateral en el seno constituyen una zona de difícil abordaje quirúrgico.

La reparación quirúrgica de fístulas de LCR ha progresado drásticamente con el desarrollo de ópticas endoscópicas y la mejora en el conocimiento de la anatomía de la base de cráneo. Los endoscopios permiten una visualización directa, localización del defecto y por ende una reparación precisa que traduce menos recidivas y menor morbilidad asociada a accesos transcraneales realizados previamente.

El propósito de este artículo es presentar el caso de paciente con fístula del receso lateral del seno esfenoidal, su reparación endoscópica y discusión del origen de la persistencia del canal de Sternberg como causa del defecto.

Palabras clave: Fístula líquido cefalorraquídeo, seno esfenoidal, canal Sternberg.
\end{abstract}

\begin{abstract}
Fístulas liquid cerebrospinal (CSF) correspond to an abnormal communication between the subarachnoid space and the nasal cavity.

The origin of lateral fístulas of the sphenoid sinus is a congenital defect of the skull base, with an incomplete or premature fusion of bone components involved in the complex process of ossification of sphenoid. This originates a channel without bone coverage, only covered by connective tissue, called channel craneofaringeo or Sternberg channel. This is a weak point where may appear encephaloceles and/or fístula, the lateral location in sinus is a difficult surgical approach.

Surgical repair of fístulas of CSF has progressed dramatically with the development of endoscopic optics and the improvement in the knowledge of the anatomy of the skull
\end{abstract}

\footnotetext{
${ }^{1}$ Médico Servicio Otorrinolaringología, Hospital Barros Luco Trudeau.
} 
base. Endoscopes enable a direct visualization, location of the defect and thus accurate reparation and less morbidity associated with transcraneales accesses made previously.

The purpose of this article is to present the case of patient with fístula of the lateral recess of the sphenoid sinus, endoscopic repair and discussion of the origin of the persistence of the channel of Sternberg as a cause of the defect.

Key words: Cerebrospinal fluid leak, sphenoid sinus, Sternberg channel

\section{INTRODUCCIÓN}

Las fístulas de líquido cefalorraquídeo (LCR) corresponden a una comunicación anómala entre el espacio subaracnoideo y la cavidad nasal o la caja timpánica. Cerca del $80 \%$ de estas fístulas se producen por traumas, el $16 \%$ atribuibles a iatrogenia o por tumores en cirugías endonasales e intracraneanas. El $4 \%$ restante se producen de forma espontánea y para ellas su diagnóstico es de exclusión de las etiologías anteriormente mencionadas ${ }^{1,2}$.

Dentro de las espontáneas, éstas pueden presentarse con y sin signos de hipertensión endocraneana (HE). Existe un grupo de pacientes que padecen de HE benigna en el cual hay hallazgos clínicos y radiológicos de hipertensión endocraneana, pero sin causa aparente.

Entre las causas congénitas tenemos los meningoceles, meningoencefaloceles, encefalocele y la persistencia del conducto de Sternberg (CS). El origen de las fístulas del CS espontáneas laterales del seno esfenoidal se encuentra en un defecto congénito de la base del cráneo, resultado de una incompleta o prematura fusión de los componentes óseos implicados en el complejo proceso de osificación del esfenoides. Ello daría lugar a un canal sin cobertura ósea, solo cubierto por tejido conectivo, que se denomina canal craneofaríngeo lateral 0 de Sternberg. Este se convierte en un punto débil donde pueden aparecer encefaloceles y/o fístulas, por su ubicación lateral en el seno constituyen una zona de difícil abordaje quirúrgico $0^{3-4}$.

Los defectos óseos que originan fístulas de LCR suelen localizarse en la región ventral de la base de cráneo, la cual incluye la lámina cribosa del etmoides, la lámina lateral, el seno esfenoidal en su línea media, el hueso temporal y la pared posterior del seno frontal ${ }^{5-6}$.

La clínica de estos pacientes es típica caracterizada por rinorrea acuosa unilateral intermitente, cuya pérdida de líquido se asocia con los cambios de posición y maniobras de exhalación con glotis cerrada. Los análisis de laboratorio pueden confirmar el diagnóstico ${ }^{7}$.

En relación a la evolución de la enfermedad la mayoría de los casos de origen traumático se resuelven con manejo conservador como reposo en cama, elevación de la cabeza y evitar actividades como toser, sonarse y la aparición de constipación. Con estas medidas y el apoyo opcional de un drenaje lumbar se suelen resolver en un plazo de $7 \mathrm{a}$ 10 días.

Por otra parte, aquellas que no se resuelven con el manejo conservador, con daño intracraneal extenso y las identificadas durante el tiempo quirúrgico de una cirugía endoscópica nasal, requieren de manejo quirúrgico oportuno, en este último ejemplo, la reparación debe ser inmediata 8 .

La reparación quirúrgica de las fístulas de LCR ha progresado drásticamente con el desarrollo de las ópticas endoscópicas y la mejora en el conocimiento de la anatomía de la base de cráneo. Los endoscopios permiten una visualización directa, la localización del defecto de forma específica y por ende una reparación precisa que se traduce en una disminución de las recidivas y la morbilidad que acompañaba a los accesos transcraneales realizados previamente.

Las fístulas que se producen a nivel del esfenoides son un real desafío, especialmente los localizados en el receso lateral, los abordajes transcraneales son excesivamente extensos e incluso usando microscopio como en abordajes transeptales no es posible visualizar la pared lateral del seno esfenoidal. Además la presencia de estructuras neurovasculares lo torna aún más complejo, por lo que se hace necesario un abordaje a través de la fosa pterigopalatina para acceder con claridad y seguridad a un receso lateral de un seno esfenoidal excesivamente neumatizado. 


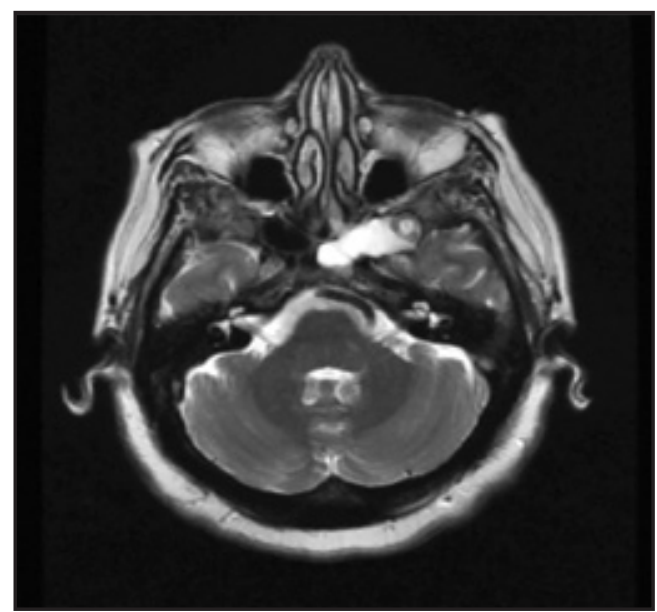

Figura 1. Resonancia magnética, T2. Evidencia sitio de ocupación del seno esfenoidal con LCR.

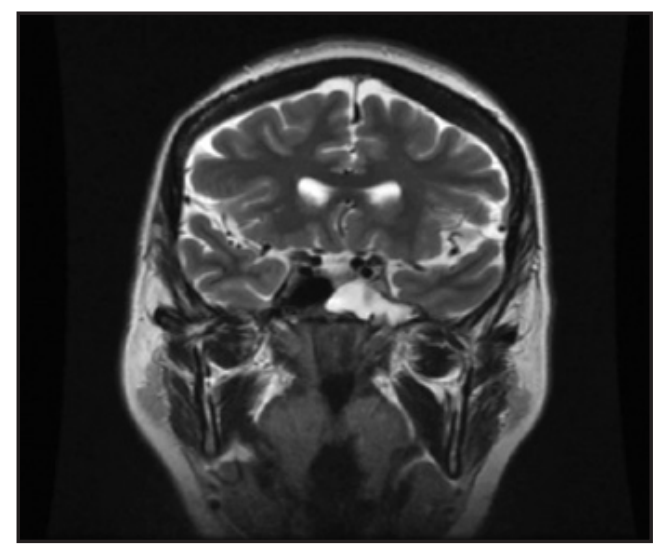

Figura 2. Resonancia magnética, corte coronal, T2. Ocupación del seno esfenoidal izquierdo con proyección hacia el receso lateral.

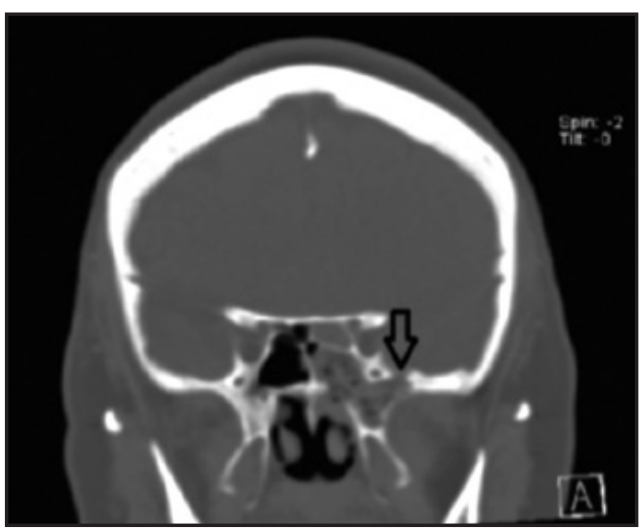

Figura 3. Tomografía computarizada, corte coronal. Ocupación del seno esfenoidal, con defecto óseo en el techo del receso lateral del seno esfenoidal izquierdo.
El propósito de este artículo es presentar el caso de paciente con una fístula de LCR del seno esfenoidal, su reparación vía endoscópica y discutir acerca del origen de la persistencia del canal de Sternberg como causa del defecto.

\section{CASO CLÍNICO}

Se presenta el caso de paciente de sexo femenino de 64 años de edad, sin antecedentes mórbidos conocidos, con historia clínica caracterizada por rinorrea acuosa unilateral por fosa nasal izquierda de 1 año de evolución, no asociada a cefalea ni a traumas previos. Se confirma diagnóstico con uso de B2 transferrina y tomografía computarizada con contraste que evidencia ocupación del seno esfenoidal.

Se complementa estudio imagenológico con resonancia nuclear magnética (RNM) que evidencia ocupación del seno esfenoidal con LCR hiperintenso en secuencia T2 de la RNM, con neumatización extensa del receso lateral y con defecto óseo en el techo de éste (Figuras 1-3).

Se realiza planificación quirúrgica con uso de fluoresceína al 5\% según lo descrito en la literatura nacional e internacional ${ }^{9-10}$, uso de injertos autólogos como son la fascia lata y grasa ${ }^{11-12}$, tiempo quirúrgico con técnica endoscópica con acceso transpterigoideo (Figuras 4-6).

Tras descongestión de fosa nasal con gasas con adrenalina y vasoconstrictor, se inicia la cirugía con el desplazamiento a medial del cornete medio. Posteriormente, se realiza antrostomía maxilar amplia, etmoidectomía completa y esfenoidotomía. La esfenoidotomía se amplía lateralmente con pinza de Kerrison y fresas. Se realiza elevación de la mucosa de la pared posterior de seno maxilar y elevación del periostio de la apófisis ascendente del hueso palatino; localización y cauterización de la artería esfenopalatina y sus ramas. Se accede a pared posterior del seno maxilar con pinza de Kerrison, con disección de medial a lateral hasta la exposición de la fascia que envuelve el tejido graso de la fosa pterigopalatina. A este nivel, la disección del tejido graso y las estructuras neurovasculares ha de ser cuidadosa, y se utiliza electrocoagulación bipolar para control de la hemorragia, la cual proviene de la arteria maxilar interna. Se realiza 
fresado cuidadoso de la lámina medial de la apófisis pterigoides para así evitar la lesión del nervio del conducto pterigoideo o nervio vidiano ubicado en el piso del seno esfenoidal y de la rama maxilar del nervio trigémino (V2). Con todo lo anterior se consigue un acceso adecuado del receso lateral del esfenoides y mejor control de la lesión, con la posibilidad de utilizar ópticas de diferentes ángulos, como son de $45^{\circ}$ y $70^{\circ}$, así como el espacio suficiente para la manipulación y reconstrucción del defecto. En este caso se reparó con multicapas. Se utilizó grasa con técnica de "tapón de bañera", fascia lata overlay y uso de material hemostático reabsorbible (Surgicel ${ }^{\circledR}$ ) para cubrir y rellenar el seno esfenoidal. No se utilizó taponamiento nasal al finalizar el procedimiento quirúrgico para evaluar eventual rinorraquia posquirúrgica inmediata.

Paciente evoluciona satisfactoriamente en posoperatorio, sin recidivas durante el periodo de seguimiento.

\section{DISCUSIÓN}

La rinorrea secundaria a una fístula de LCR es una condición de cuidado dada la comunicación, entre el espacio subaracnoideo y la cavidad nasal, la que posee una flora bacteriana residente que puede ser la puerta de entrada para el desarrollo de una infección endocraneana. Distintas series reportan que el $80 \%$, incluso más, de los pacientes portadores de una fístula de LCR presentaron una meningitis bacteriana, siendo ésta más frecuente en aquellos con etiología traumática como causa del defecto ${ }^{13-15}$

Dado que la rinorrea es un signo clínico inespecífico, la presencia de LCR en una muestra debe ser confirmada antes de intentar cualquier opción terapéutica, si es que la historia no orienta a esta sospecha diagnóstica. Una vez confirmada la fístula de LCR ésta debe ser loca-

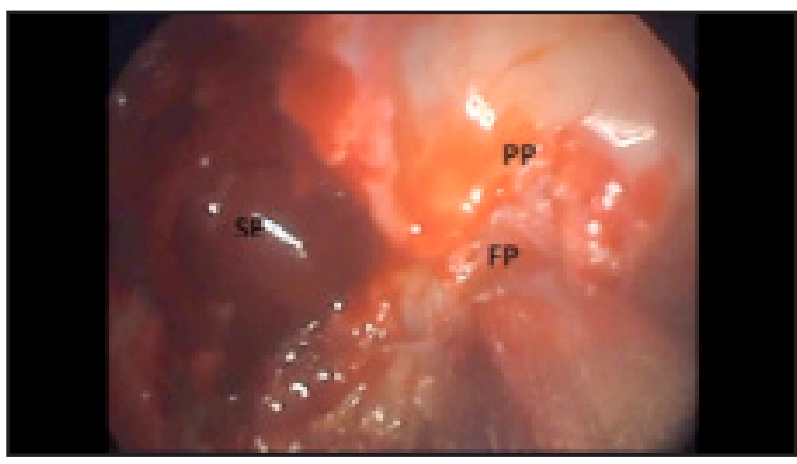

Figura 4. PP: Pared posterior seno maxilar. SE: Seno esfenoidal. FP: Fosa pterigomaxilar (óptica $0^{\circ}$ ).

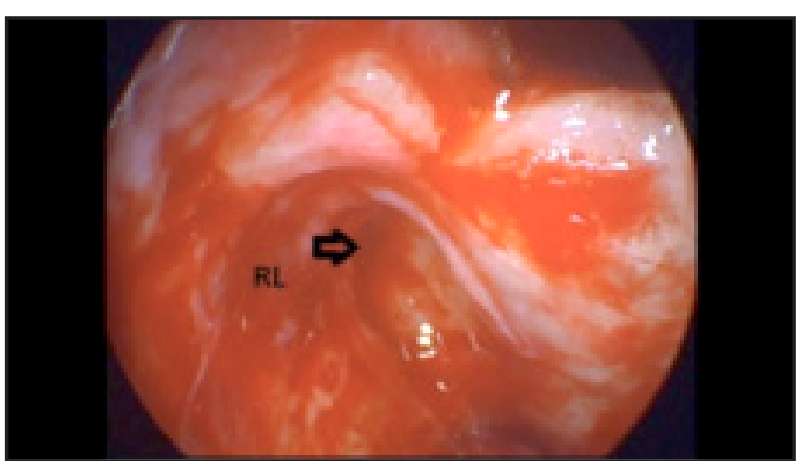

Figura 5. RL: Receso lateral esfenoides. Flecha: Defecto óseo en el techo del receso lateral (óptica $70^{\circ}$ ).

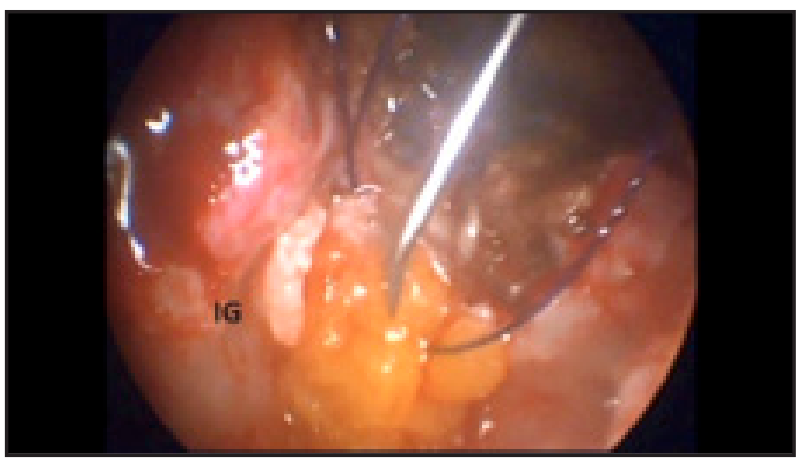

Figura 6. IG: Injerto grasa en defecto óseo del esfenoides (óptica $70^{\circ}$ ).

lizada para su reparación. Comúnmente existen distintos métodos diagnósticos que nos pueden confirmar nuestra sospecha, los más utilizados son el test de glucosa con cinta reactiva, beta 2 transferrina, entre otras. La beta 2 transferrina 
es el método más conveniente por su costo, alta sensibilidad y especificidad en el diagnóstico ${ }^{16-17}$. La beta trace proteína es otro método diagnóstico, siendo más sensible y específica pero con un mayor costo y escasa disponibilidad incluso en USA. El sitio del defecto fistuloso puede ser localizado usando estudios de imágenes como la TC de alta resolución, la resonancia magnética la cual además es útil cuando se sospecha un meningocele 0 meningoencefalocele, TC cisternografía y la prueba con fluoresceína intratecal utilizada durante la cirugía. Con excepción de la TC de alta resolución, los otros métodos de estudio de imágenes pueden simultáneamente confirmar y ubicar el sitio de fístula de $\mathrm{LCR}^{18}$. Todas estas opciones de estudio obviamente tienen distintos costos y rangos de invasividad que deben ser valorados por el tratante y conversados con el paciente.

Como hemos visto las localizaciones más frecuentes de fístulas de LCR en la base de cráneo se producen en el etmoides, ya sea, en la lámina cribosa o lamela lateral, sin embargo, aunque infrecuentes, las fístulas con origen en el esfenoides y específicamente las ubicadas en el receso lateral conllevan un desafío terapéutico mayor dado el difícil acceso durante la cirugía de reparación.

La ubicación más común de fístulas de líquido cefalorraquídeo asociada a encefaloceles es un receso lateral esfenoidal neumatizado, lateral a la segunda rama del trigémino más conocida como V2. A pesar de signos y síntomas de hipertensión endocraneana en estos individuos los cuales concuerdan con el origen espontáneo, existen autores que han propuesto un origen congénito como causa de esta patología ${ }^{12,19}$. La embriología del hueso esfenoides sugiere que la fusión del ala esfenoidal, la base, el techo y los centros de osificación existen cerca del receso lateral del seno esfenoidal. En 1988, Sternberg ${ }^{20}$, propuso la existencia de canal lateral craneofaríngeo como falla de la fusión y causa de estos defectos entre la cavidad nasal y el endocraneo. Originalmente se describió que el canal se extendía desde la unión del cuerpo del esfenoides y la raíz posterior del ala menor del esfenoides, medial a la fisura orbitaria superior (FS) y que inferiormente comunica con la nasofaringe cuando existe suficiente neumatización hacia la pared lateral del seno esfenoidal. Por definición y de acuerdo al desarrollo embriológico, este canal debe situarse medial a la FS y por lo tanto medial al foramen rotundum y a V2. Esta localización del canal de Sternberg en estudios anatómicos es inconsistente con la mayoría de las fístulas del receso lateral del esfenoides, las cuales por lo general se han ubicado lateral a V2. Además, un encefalocele originado a través de este canal teórico debería no solo atravesar el seno cavernoso sino también penetrar las dos capas de duramadre antes de pasar por la base de cráneo. La causa más probable de origen de estas lesiones es la combinación de una neumatización extensa del receso lateral esfenoidal y el desarrollo de defectos aracnoideales en el hueso que remodelan este último por efecto de hipertensión endocraneana ${ }^{20-21}$. Esta teoría fue recientemente apoyada por Schuknecht y col, los que encontraron estos defectos aracnoidales en siete de ocho fístulas de receso lateral con rinorrea espontánea22.

Para tratar con éxito las fístulas de LCR del receso lateral del esfenoides, se requiere un buen estudio preoperatorio que permita precisar su localización. En comparación con los abordajes externos de fosa media, actualmente se considera que las técnicas endoscópicas suponen la mejor opción de acceso para el tratamiento de estos defectos, por ser menos invasivas y por su alta efectividad. Lesiones en la línea media de esfenoides pueden ser reparadas con éxito con las tradicionales vías endoscópicas, transnasal-transesfenoidal 0 transetmoidoesfenoidal. Sin embargo, para aquellas situadas en la región lateral suelen ser insuficientes por las limitaciones de acceso, y el abordaje transpterigoideo ha demostrado ser el más adecuado. Cabe recordar que para acceder a la porción más lateral del seno esfenoidal, la vía debe ser a través de la pared posterior del seno maxilar y la fosa pterigopalatina, permitiendo la utilización de endoscopios de diferentes ángulos para exponer el defecto adecuadamente y manejar el material necesario para reparar la lesión y la base del cráneo. Este abordaje conlleva un aumento de potenciales complicaciones intraoperatorias neurovasculares, por la presencia de la arteria maxilar interna y sus ramas, con un alto flujo dada la afluencia del sistema carotideo externo, el nervio maxilar en su segunda rama (V2) y el nervio vidiano. No obstante, son complicaciones evitables si se realiza una disección cuidadosa separando lateralmente 
el contenido de la fosa pterigopalatina y con una adecuada preparación para una hemostasia prolija en caso de sangrado. La hipoestesia asociada 0 no a dolor en el área inervada por V2, así como presentar ojo seco por probable daño del nervio vidiano pueden ser secuelas de la intervención, como aconteció con la paciente del caso antes presentado la cual desarrolló una hipoestesia del territorio inervado por la segunda rama del trigémino sin mayores repercusiones.

La utilización de fluoresceína intratecal al 5\%, como método de diagnóstico o su uso intraoperatorio para la localización del defecto y la comprobación del cierre de la fístula durante la cirugía, ha sido de gran ayuda. Sin embargo, se han descrito complicaciones mayores aunque sumamente infrecuentes con su uso como convulsiones y anafilaxia.

Se ha descrito una gran variedad de materiales para la reparación de las fístulas de líquido cefalorraquídeo, incluyendo materiales sintéticos, y orgánicos como fascia temporal y fascia lata, hueso, grasa y cartílago. Además, puesto que una elevada presión intracraneal contribuye a la aparición de fístula, una reconstrucción segura con una técnica multicapas tiene mayores posibilidades de resistencia.

\section{BIBLIOGRAFÍA}

1. Trichy N, Vijayalakshmi S, Palak P. Endoscopic Endonasal Repair of Sphenoid Sinus Cerebrospinal Fluid Leaks: Our Experience. Indian J Otolaryngol Head Neck Surg 2015; 67(4): 412-6.

2. Bradford A, Woodwortha J, Palmer N. Spontaneous cerebrospinal fluid leaks. Neck Surgery2009; 17 : 59-65.

3. Tomaszewska M, Brozek-Madry E, Krzeski A. Spontaneous sphenoid sinus cerebrospinal fluid leak and meningoencephalocele - are they due to patent Sternberg's canal? Videosurgery Miniinv 2015; 10 (2): 347-58.

4. Abarca A, Pardo J, Lemp M, Stott C. Reparación endoscópica endonasal de fístulas de líquido cefalorraquídeo no quirúrgicas: Experiencia Hospital Clínico Universidad de Chile. Rev
Algunos autores describen la obliteración del seno esfenoidal con grasa abdominal como única técnica de reparación para sellar el defecto. Sin embargo, si no se asocia a la resección de la mucosa adyacente al defecto óseo y la reparación de la base del cráneo mediante reconstrucción con diferentes capas, existirá un mayor riesgo de persistencia de fístula de líquido cefalorraquídeo, aparición de quistes aracnoideos y formación de mucoceles intrasinusales tardíos.

\section{CONCLUSIÓN}

Las fístulas de LCR ubicadas en el receso lateral del seno esfenoidal son originadas no solo por la persistencia de un defecto óseo como lo sería el CS, sino más bien por una combinación de factores donde la presión intracraneal elevada y la formación de "nichos" de la aracnoides que remodelan el hueso tienen un rol de suma importancia.

Sin embargo, no debemos descuidar el propósito único de la terapia de estos pacientes que son corregir el defecto anatómico con éxito, disminuyendo las persistencias y recidivas, para así evitar las temidas complicaciones infecciosas endocraneales.

Otorrinolaringol Cir Cabeza Cuello 2010; 70 : 205-14.

5. Daza C, Vargas S, Aponte E, Hernández N, Hernández C. Rinorrea de LCR de origen espontáneo. A propósito de un caso. Acta Otorrinolaringol Esp 2000; 12-N¹-2000.

6. Schmidt T, Rebolledo V, Kawaguchi K, Santamaría A, Pinto J. Abordaje endoscópico de las fístulas de líquido cefalorraquídeo. Rev Otorrinolaringol Cir Cabeza Cuello 2003; 63: 112-6.

7. Oakley gM, Alt Ja, Schlossce RJ, Harvey RJ, OrLandI RR. Diagnosis of cerebrospinal fluid rhinorrhea: an evidence-based review with recommendations. International Forum of Allergy \& Rhinology 2016; №1: 8-16.

8. Mao V, Keane W, Atkins J, Spiegel J, Willcox T, Rosen M, Andrews D, Zwillenberg D. Endoscopic repair of cerebrospinal fluid rhinorrhea. Otolaryngol Head Neck Surg 2010; 122(1): 56-60. 
9. Schmidt T, Rebolledo V. Uso de fluoresceína en el diagnóstico y tratamiento de las fístulas de líquido cefalorraquídeo. Rev Otorrinolaringol Cir Cabeza Cuello 2003; 63: 143.

10. Kluajic V, Vulekovic P, Vlaski L, Savovic S, Dragicevic D, Papic V. Endoscopic repair of cerebrospinal fluid fístulas in nasal liquorrhea. Braz J Otorhinolaryngol 2016. http://dx.doi. org/10.1016/j.bjorl.2016.04.024.

11. Ariasa A, Bernal-Sprekelsenb M, Rioja E, Ensẽ natc J, Prats-Galinod A, Lobidb I. Abordaje endoscópico transpterigoideo y reparación de base de cráneo tras resección de meningoencefalocele esfenoidal. Nuestra experiencia. Acta Otorrinolaringol Esp 2015; 66(1): 1-7.

12. Castelnuovo P, Dallan I, Pistochini A, Battaglia P, Locatell D, Bignami M. Endonasal endoscopic repair of Sternberg's canal cerebrospinal fluid leaks. Laryngoscope 2007; 117: 345-9.

13. Sharma DP, Singh D, Sinha S, Srivastva AK, Singh H, JAGETIA A, ET AL. CSF rhinorrhea: an overview of endoscopic repair. Indian J Neurotrauma 2010; 7: 157: 62-13.

14. Crawford $C$, Kennedy N, Weir WR. Cerebrospinal fluid rhinorroeaand Hemophilus influenzae meningitis 37 years after a headinjury. J Infect 1994; 28: 9: 7-14.

15. Dullvesi D, Vulekovic P, Cigic T, Kojadinovic Z, Papic V, Horvat I. Post-traumatic communication of endocranium and nasal cavity with recurrent meningoencephalitis without rhinorrhea. Case report. Vojnosanitetski pregled 2010; 67: 945-8.
16. Warnecke A, Averbeck T, Wurster U, Harmening M, Lenarz T, Stover T. Diagnostic relevance of beta2transferrin for the detection of cerebrospinal fluid fístulas. Arch Otolaryngol Head Neck Surg 2004; 130: 1178-84.

17. Gorogh T, Rudolph P, Meyer JE, Werner JA, Lippert BM, Maune S. Separation of beta2-transferrin by denaturing gel electrophoresis to detect cerebrospinal fluid in ear and nasal fluids. Clin Chem 2005; 51: 1704-10.

18. Reddy M, Baugnonlmaging K. Imaging of Cerebrospinal Fluid Rhinorrhea and Otorrhea. Radiol Clin N Am 2017; 55: 167-87.

19. Schick B, Brors D, Prescher A. Sternberg's canal - cause of congenital sphenoidal meningocele. Eur Arch Otorhinolaryngol 2000; 257: 430-2.

20. Reynolds JM, Tomkinson A, Grigg RG, et al. A Le Fort I osteotomy approach to lateral sphenoid sinus encephalocoeles. J Laryngol Otol 1998; 112: 779-81.

21. Lal SY, Kennedy DW, Bolger WE. Sphenoid encephaloceles: disease management and identification of lesions within the lateral recess of the sphenoid sinus. Laryngoscope 2002; 112 : 1800-5.

22. Schuknecht B, Simmen D, Briner HR, et al. Nontraumatic skull base defects with spontaneous CSF rhinorrhea and arachnoid herniation: imaging findings and correlation with endoscopic sinus surgery in 27 patients. AJNR Am J Neuroradiol 2008; 29: 542-9. 